\section{Tradução e adaptação da versão brasileira do Night Eating Questionnaire}

\author{
Translation and adaptation of the Brazilian version \\ of the Night Eating Questionnaire
}

${ }_{1}$ Curso de Nutrição, Universidade do Vale do Rio dos Sinos, São Leopoldo, Brasil.

2 Serviço de Anestesia e Medicina Perioperatória, Hospital de Clínicas de Porto Alegre, Porto Alegre, Brasil. ${ }^{3}$ Instituto de Ciências Básicas da Saúde, Universidade Federal do Rio Grande do Sul, Porto Alegre, Brasil.

4 Faculdade de Medicina, Universidade Federal do Rio Grande do Sul, Porto Alegre, Brasil.

Correspondência M. P. L. Hidalgo

Departamento de Psiquiatria e Medicina Legal, Faculdade de Medicina, Universidade Federal do Rio Grande do Sul. Rua Castro Alves 167, sala 204, Porto Alegre, RS 90430-131, Brasil. mpaz@cpovo.net

\section{Abstract}

The goal of this study was to translate and adapt the Night Eating Questionnaire to Portuguese and assess the reliability of the Portuguese-language version. The study included two phases. The first consisted of: (1) translation into Portuguese; (2) back-translation into English; (3) correction and semantic adaptation; (4) content validation; and (5) assessment of the understanding of the questionnaire by means of $10 \mathrm{~cm}$ Visual Analogue Scales with 30 adult users of a nutritional support clinic. In the second phase, the questionnaire's reliability was assessed in 100 subjects with similar demographic characteristics to the first sample. In the fifth step, understanding of the instrument assessed by the visual analogue scales was $8.20 \pm 1.55 \mathrm{~cm}$. The instrument showed satisfactory internal consistency, with an overall Cronbach $\alpha$ of 0.78. The Portuguese version proved to be easily understandable, with good semantic validation and consistency, suggesting that the questionnaire may be a good instrument for screening the night eating syndrome. However, other psychometric characteristics of this instrument need to be assessed in samples with different social and educational levels.

Circadian Rhythm; Chronobiology; Food Habits
Ana Beatriz Cauduro Harb 1

Wolnei Caumo 2,3

Maria Paz Loayza Hidalgo 4

\section{Introdução}

Doenças cardiovasculares coronarianas, dislipidemias, hipertensão, obesidade e diabetes mellitus formam um conjunto de morbidades geralmente associadas entre si, constituindo-se em graves problemas de saúde pública 1,2 . Numerosos estudos demonstraram que na etiologia dessas doenças encontram-se fatores cujas etiologias podem estar vinculadas às características genéticas, estilo e qualidade de vida. O último é caracterizado pelo modo de viver, que conduz a práticas, hábitos, pressões e desgaste físico-psicológico, que se constituem em fatores de risco, tais como tabagismo, etilismo, alimentação inadequada, sedentarismo e estresse 3 . Pesquisas têm mostrado que além do tipo e quantidade de alimentos ingeridos, o impacto dos hábitos alimentares no processo saúde-doença é também influenciado pelo modo de alocação da alimentação no curso do dia 4,5,6,7,8.

O comportamento alimentar é complexo, envolvendo aspectos metabólicos, fisiológicos e ambientais 9,10, que apresentam ritmicidade circadiana, herdada e espécie específica, sendo a humana essencialmente diurna. Está sincronizado ao ciclo claro/escuro 10,11,12 e aos níveis de cortisol, serotonina, leptina, citocinas, entre outros 10,12,13. Além disso, o ritmo social imprime marcado efeito na regulação da alimentação, pois a sociedade contemporânea funciona 24 horas e seu impacto na quantidade, qualidade 
e horários da alimentação tem sido inexorável. Como exemplo, cita-se o atraso da alimentação para adaptação aos horários de trabalho ${ }^{11}$.

Dada a relevância de investigar de maneira sistemática os hábitos alimentares, pesquisadores perceberam que perguntas isoladas sobre os hábitos de comer à noite não avaliavam de modo acurado o "comer noturno". Então, a partir dos estudos de Birketvedt et al. 14,15 surgiram pesquisas com o objetivo de sistematizar a investigação dos hábitos do comer noturno 14, que constituem uma síndrome, caracterizada como um atraso circadiano do padrão alimentar 16, mediado por alterações neuroendócrinas ao estresse 14,17,18. Dentre os critérios diagnósticos dessa síndrome relacionam-se: anorexia matutina; ingestão $\geq 50 \%$ da energia diária após às 19:00; despertar para comer ao menos uma vez por noite nos últimos três meses, com consciência do ato; consumo freqüente de lanches de alto valor calórico nos despertares noturnos; ausência de critérios para bulimia nervosa ou "binge eating disorder" 14.

Sua prevalência em clínicas de obesidade é de $8,9 \%$ a $12 \%$ e de até $27 \%$ em pacientes que procuram cirurgia para obesidade 18 . O reconhecimento e tratamento dessa síndrome tem crescido e parece caminho promissor para o tratamento da obesidade 19, que se constituiu em problema de saúde pública em diversos países, incluindo o Brasil. Portanto, a avaliação de fatores de risco para a obesidade deve priorizar o desenvolvimento de métodos sistematizados que permitam rastrear hábitos de alimentação implicados no seu curso, ainda pouco explorados na pesquisa e na clínica, como a alocação dos alimentos no turno da noite. Sobretudo, a avaliação desse hábito se reveste de importância ainda maior frente aos hábitos da sociedade moderna, que não privilegia espaços para alimentação adequada durante a rotina de trabalho 11 . Com o intuito de padronizar o diagnóstico e facilitar comparações entre estudos clínicos sobre o tema, em 2004 foi criado o Night Eating Questionnaire (NEQ) 20. Esse instrumento foi desenvolvido em Língua Inglesa, no entanto, para aplicação à população brasileira o mesmo deve ser traduzido, adaptado e validado. Assim, o presente estudo foi realizado em duas fases. A primeira, com o objetivo de traduzir e adaptar o conteúdo do NEQ de 15 itens para uma versão em Português, denominado Questionário sobre Hábitos do Comer Noturno. A segunda, com o objetivo de avaliar a confiabilidade do novo instrumento.

\section{Sujeitos e métodos}

Este estudo, com delineamento transversal, envolveu duas fases. A primeira constituiu-se da seguinte forma: (i) tradução; (ii) retradução para o Inglês; (iii) correção e adaptação da semântica; (iv) validação do conteúdo e; (v) avaliação da clareza do questionário por meio de escalas analógico-visuais de $10 \mathrm{~cm}$. A avaliação da clareza do questionário envolveu trinta sujeitos selecionados de maneira aleatória com base em uma lista de pacientes atendidos em clínica de suporte nutricional. Foram incluídos apenas sujeitos com nível de escolaridade acima de 11 anos que deram seu consentimento escrito livre e esclarecido. $\mathrm{O}$ estudo observou os princípios éticos envolvidos na pesquisa em seres humanos e foi aprovado pelo Comitê de Ética da Universidade Federal do Rio Grande do Sul. Na segunda fase do estudo, avaliou-se a confiabilidade aplicando o instrumento em 100 sujeitos da mesma clínica de suporte nutricional, obedecendo aos mesmos critérios de inclusão e princípios éticos utilizados na primeira fase deste estudo.

\section{$\underline{\text { Instrumentos }}$}

\section{- Avaliação do perfil sócio-demográfico}

O perfil sócio-demográfico foi avaliado por meio de questionário estruturado com o objetivo de avaliar o nível de escolaridade e as características da amostra.

\section{- Night Eating Questionnaire}

Trata-se de um questionário composto de 14 questões do tipo likert. Cada questão apresenta cinco possibilidades de resposta (0-4). O escore total varia de 0 a 56 . Maiores escores correspondem à maior probabilidade de apresentar a síndrome do comer noturno. A 15a questão se relaciona ao tempo em que vem tendo problemas com a alimentação noturna. A resposta dessa questão não entra na composição do escore da escala, embora seja um descritor do curso da síndrome 20 .

\section{Descrição das fases do estudo}

\section{- Fase de tradução, adaptação e validação de conteúdo semântico}

Buscando alcançar o maior grau de equivalência semântica entre instrumentos aplicados em idiomas diferentes, a tradução e adaptação da versão original em Inglês foram baseadas nas recomendações do manual para o processo de 
adaptação de medidas de auto-relato em diferentes culturas ${ }^{21}$. Utilizou-se o processo de tradução e versão (forward and backtranslation) usado na tradução e adaptação cultural de instrumentos amplamente empregados como o questionário sobre saúde e qualidade de vida - a Escala de Compulsão Alimentar Periódica 22 - e nos trabalhos de equivalência semântica das versões em Português dos instrumentos Childhood Trauma Questionnaire 23 e Abuse Assessment Screen 24. As quatro etapas do processo de tradução e adaptação do Questionário sobre Hábitos do Comer Noturno estão apresentadas na Tabela 1.

A primeira etapa consistiu na tradução do instrumento original do idioma Inglês para o Português, realizada por um lingüista profissional, graduado em Letras, com especialização em Língua Inglesa e experiência na área temática (hábitos alimentares). Na segunda etapa, a primeira versão em Língua Portuguesa foi novamente traduzida para o Inglês por uma nutricionista e tradutora bilíngüe, cujo idioma nativo é o Inglês. A terceira etapa foi a revisão técnica e a equivalência semântica realizadas, independentemente, por três profissionais: um professor de Língua Portuguesa com especialização em Inglês e uma nutricionista bilíngüe com ampla experiência em avaliação clínica nutricional. Também participou dessa etapa uma pesquisadora bilíngüe, da área neuro-cognitiva, com experiência em criação e adaptação de escalas e testes diagnósticos. Nessa etapa, priorizaram-se duas questões: a equivalência semântica entre a primeira e a segunda traduções, sob a perspectiva do significado referencial dos termos/palavras constituintes. A outra questão foi o significado geral de cada pergunta, instrução ou opção de resposta do instrumento captado na tradução em relação ao original.

O significado referencial representa as idéias que uma única palavra ou conjunto de palavras aludem. Presume-se que, se o significado referencial é o mesmo no original e na tradução, existe uma correspondência literal entre eles 25 .

Por outro lado, o significado geral transcende a literalidade dos termos e assertivas e considera os aspectos culturais da população alvo. O objetivo é avaliar a pertinência, adequação e aceitabilidade do estilo empregado ou o uso específico de um termo nos itens do instrumento. Dessa maneira o significado geral é muito importante em virtude da tradução literal de uma palavra não implicar, necessariamente, a mesma evocação subjetiva em diferentes culturas. Questões como os níveis educacional e sócio-econômico são variáveis que devem ser levadas em consideração numa adaptação como a presente 26 .
Os três profissionais supracitados compararam o instrumento traduzido e apontaram algumas modificações e correções, objetivando adequar à versão traduzida os significados referencial e geral, concluindo-se numa versão corrigida da escala (Tabela 1).

Na quarta etapa, para avaliar a validação do conteúdo e do significado geral dentro do contexto da população-alvo, a versão corrigida foi apresentada para cinco profissionais de saúde que trabalham com nutrição clínica. Outras cinco pessoas não relacionadas com essa área (estudantes universitários) também foram consultadas. Foi solicitado que lessem o instrumento e sugerissem modificações se a linguagem não parecesse adequada, anotando a razão para essa observação. A partir das sugestões foram feitas modificações no instrumento para uma versão final (Tabela 2), sendo escolhidos e incorporados itens oriundos das etapas anteriores ou optando por certas modificações para melhor atender os critérios expostos mencionados.

Na quinta etapa, a versão final foi aplicada para trinta sujeitos adultos atendidos em clínica de suporte nutricional. A média de escolaridade em anos completos foi de $14,48 \pm 2,98$. Os usuários avaliaram a clareza de cada uma das 15 questões da versão traduzida e adaptada para o Português brasileiro do Questionário sobre Hábitos do Comer Noturno por meio da escala analógico-visual de $10 \mathrm{~cm} 27,28$. A questão norteadora era: "avalie a clareza da questão". Zero correspondia a "nada claro" e 10 "muito claro". Foram definidos como indicador de compreensão insuficientes valores abaixo do percentil 25. A compreensão global do instrumento foi definida como a média aritmética das 14 questões que compõem o escore do Questionário sobre Hábitos do Comer Noturno.

\section{- Fase de avaliação da confiabilidade do Questionário sobre Hábitos do Comer Noturno}

A segunda fase do estudo teve a finalidade de medir a confiabilidade da escala por meio do coeficiente alfa de Cronbach, que mede a homogeneidade dos componentes da escala, ou seja, a consistência interna dos 14 itens que compõem o escore final do instrumento. Para isso a escala foi respondida por 100 sujeitos com escolaridade acima de 11 anos.

\section{Análise estatística}

As informações coletadas foram organizadas no programa SPSS versão 12.0 (SPSS Inc., Chicago, Estados Unidos). A análise exploratória dos dados constou de testes estatísticos descritivos e 
Avaliação da equivalência semântica entre o instrumento (Night Eating Questionnaire) original em Inglês, a versão traduzida para o Português, a versão novamente traduzida para o Inglês e a versão final em Português.

Original
1. How hungry are you usually in the
morning?
2. When do you usually eat for first
time?
3. Do you have cravings or urges to
eat snacks after supper, but before
bed time?
4. How much control do you have
over your eating between supper
and bed time?
5. How much of your daily food
intake do you consume after supper
time?

7. When you are felling blue, is your mood lower in the:

8. How often do you have trouble getting to sleep?

9. Other than only to use the bathroom, how often do you get up at least once in the middle of the night?

If 0 on question 9, please stop here

10. Do you have cravings or urges to eat snacks when you wake up at night?

11. Do you need to eat in order to get back to sleep when you awake at night?

12. When you get up in the middle of the night, how often do you snack?

If 0 on question 12, please skip to question 15
Como é usualmente seu apetite de manhã?

Quando você usualmente come pela primeira vez no dia?

Você tem compulsões "cravings" para fazer lanches depois da janta, mas antes de deitar?

Quanto você consegue controlar sua alimentação entre a janta e a hora de ir deitar?

Quanto da sua ingestão diária (alimentos) você consome depois de jantar?

Você costumeiramente sente-se triste, deprimido, sentindo-se um lixo?

Quando você sente-se triste, seu humor é pior no(a):

Quantas vezes você tem problemas para pegar no sono?

Quantas vezes você acorda ao menos uma vez no meio da noite, (por outra razão que não seja usar o banheiro)

Se o escore estiver 0 na questão 9, parar aqui

Você tem compulsões ("cravings") para fazer lanches quando acorda à noite?

Quando você se acorda à noite, você necessita comer para voltar a dormir?

Quando você se acorda na metade da noite, quantas vezes você lancha?

Se o escore for 0 na 12, passe para a pergunta 15

\section{Retraduzida para Inglês}

Versão final

How is your appetite in the morning Normalmente, como é o seu apetite de manhã?

When do you usually for the first time in a day?

Do you have any cravings to have snacks after dinner, but before going to bed?

Can you control your eating between diner and bed time?

How much of your daily ingestion of food do you consume after dinner?

Do you usually feel sad, depressed, like trash?

When you feel sad, your mood is at worst:

How often do you have problems to fall asleep?

How often do you wake up at the least once in the middle of the night (for any other reason than going to the bathroom)?

\section{If you have scored 0 in the question 9, stop here}

Do you have any cravings to have snacks when you wake up at night?

When you wake up at night, do you need to eat to go back to sleep?

When you wake up in the middle of the night, how often do you have a snack?

If you score 0 for number 12, go on to question 15
Normalmente, quando você come pela primeira vez ao dia?

Depois do jantar você tem compulsões ou desejos de fazer lanches antes de deitar?

O quanto você consegue controlar a sua alimentação entre a janta e a hora de ir deitar?

Em relação à sua ingestão diária de alimentos, quanto você consome depois do jantar?

Você costumeiramente se sente triste, deprimido ou um lixo?

Quando você se sente triste, em qual parte do dia seu humor é pior?

Com que freqüência você tem dificuldades para pegar no sono?

Quantas vezes você acorda pelo menos uma vez no meio da noite (por outra razão que não seja usar o banheiro)?

\section{Se o escore for 0 na pergunta 9, pare aqui}

Você tem compulsões ou desejos de fazer lanches quando acorda à noite?

Quando você acorda à noite, você precisa comer para voltar a dormir?

Quando você acorda no meio da noite, quantas vezes você lancha?

Se for 0 na pergunta 12, passe para a pergunta 15

(continua) 
Tabela 1 (continuação)

\begin{tabular}{|c|c|c|c|}
\hline Original & Traduzida & Retraduzida para Inglês & Versão final \\
\hline $\begin{array}{l}\text { 13. When you snack in the middle of } \\
\text { the night, how aware are you of your } \\
\text { eating? }\end{array}$ & $\begin{array}{l}\text { Quão consciente você está quando } \\
\text { você lancha no meio da noite? }\end{array}$ & $\begin{array}{c}\text { How conscious are you when you } \\
\text { have a snack in the middle of the } \\
\text { night? }\end{array}$ & $\begin{array}{c}\text { Qual o seu nível de consciência } \\
\text { quando você lancha no meio da } \\
\text { noite? }\end{array}$ \\
\hline $\begin{array}{l}\text { 14. How much control do you have } \\
\text { over your eating while you are up } \\
\text { at night? }\end{array}$ & $\begin{array}{l}\text { Quanto controlado você está sobre } \\
\text { sua alimentação enquanto você está } \\
\text { acordado à noite? }\end{array}$ & $\begin{array}{l}\text { How much are you in control of your } \\
\text { eating while you are awake at night? }\end{array}$ & $\begin{array}{c}\text { Qual o nível de controle sobre sua } \\
\text { alimentação enquanto você está } \\
\text { acordado à noite? }\end{array}$ \\
\hline $\begin{array}{l}\text { 15. How long have your current } \\
\text { difficulties with night eating been } \\
\text { going on? }\end{array}$ & $\begin{array}{l}\text { Há quanto tempo você vem tendo } \\
\text { dificuldades com a alimentação } \\
\text { noturna? }\end{array}$ & $\begin{array}{c}\text { How long have you had difficulty in } \\
\text { eating in the night? }\end{array}$ & $\begin{array}{c}\text { Há quanto tempo você vem tendo } \\
\text { dificuldades com a alimentação } \\
\text { noturna? }\end{array}$ \\
\hline
\end{tabular}

Tabela 2

Versão final do Questionário sobre Hábitos do Comer Noturno com as médias e desvio-padrão (DP) do grau de clareza de cada questão medido pela escala analógico-visual $(\mathrm{n}=30)$.

\begin{tabular}{lcc}
\hline Questões & Média (cm) & DP \\
\hline 1. Normalmente, como é o seu apetite de manhã? & 9,24 & 1,09 \\
2. Normalmente, quando você come pela primeira vez ao dia? & 9,30 & 0,79 \\
3. Depois do jantar, até a hora de dormir, você tem compulsões ou desejos de fazer lanches? & 9,26 & 1,02 \\
4. O quanto você consegue controlar a sua alimentação entre a janta e a hora de ir deitar? & 9,41 & 0,74 \\
5. Em relação à sua ingestão diária de alimentos, qual a quantidade que você costuma consumir depois de jantar? & 8,92 & 1,47 \\
6. Você costumeiramente se sente triste, deprimido ou um lixo? & 9,01 \\
7. Quando você se sente triste, em qual parte do dia seu humor é pior: & 9,21 \\
8. Com que freqüência você tem dificuldades para pegar no sono? & 9,91 \\
9. Quantas vezes você acorda pelo menos uma vez no meio da noite (por outra razão que não seja usar o banheiro)? & 8,36 \\
Se o escore for 0 na pergunta 9, pare aqui & 0,79 \\
10. Você tem compulsões ou desejos de fazer lanches quando acorda à noite? & 1,85 \\
11. Quando você acorda à noite, você precisa comer para voltar a dormir? & 9,34 \\
12. Quando você acorda no meio da noite, quantas vezes você lancha? & 9,28 \\
Se o escore for 0 na pergunta 12, passe para a pergunta 15 & 8,89 \\
13. Qual o seu nível de consciência quando você lancha no meio da noite? & 0,89 \\
14. Qual o nível de controle sobre sua alimentação enquanto você está acordado à noite? & 1,54 \\
15. Há quanto tempo você vem tendo dificuldades com a alimentação noturna? & 8,96 \\
\hline
\end{tabular}

de freqüências 29 . Para verificar a consistência interna do Questionário sobre Hábitos do Comer Noturno foi utilizado o coeficiente de fidedignidade de Cronbach 30 .

\section{Resultados}

As etapas da tradução e validação de conteúdo estão apresentadas na Tabela 1. O instrumento final encontra-se na Tabela 2, na qual também são apresentados as médias e desvios-padrão para o nível de clareza de cada questão.
Após as terceira e quarta etapas, algumas alterações foram necessárias na geração da versão final. Nas questões 1 e 2, o termo "usually" tem sua tradução literal como habitualmente, optouse pelo termo normalmente, que também pode ser um significado do termo em Inglês, pois, na opinião dos profissionais da etapa 4 , teria menor probabilidade de gerar dúvida. Na questão oito, a expressão "how often" foi substituída por "com que freqüência”, pois a tradução literal "quantas vezes" poderia dificultar a compreensão da pergunta, que visa a obter informação sobre qual a freqüência que apresenta dificuldade para dor- 
mir. Na questão 10, o termo “cravings", predominantemente médico e de pouco uso pela população, que na tradução literal significa desejo, poderia não expressar o significado de um desejo compulsivo. Dessa forma, optou-se por utilizar na versão final "desejo ou compulsão".

Os resultados obtidos na etapa 5 do procedimento metodológico evidenciaram que para todas as questões do Questionário sobre Hábitos do Comer Noturno, as médias de compreensão avaliadas pela clareza das questões na escala analógico-visual foram acima de 7,6 cm (Tabela 2). A avaliação de compreensão total teve média de $8,20 \pm 1,55 \mathrm{~cm}$.

Os itens 9 (razão do despertar que não fosse para usar o banheiro), 12 (número de lanches feitos à noite), 13 (nível de consciência enquanto está fazendo lanche à noite) e 15 (tempo que vem tendo dificuldade com a alimentação noturna) tiveram a menor média de compreensão, evidenciada pela maior dispersão nas médias das respostas (Tabela 2).

A avaliação da confiabilidade do Questionário sobre Hábitos do Comer Noturno por meio do coeficiente alfa de Cronbach demonstrou um coeficiente geral de 0,78 , que aumentou para 0,82 ao ser retirada a questão número 7 (Quando você se sente triste, em qual parte do dia seu humor é pior?) (Tabela 3).

\section{Discussão}

Neste estudo se traduziu e adaptou o NEQ. Também, foi verificada a confiabilidade do instrumento. O NEQ foi construído no idioma Inglês para screening clínico sobre a síndrome do comer noturno na população americana. No entanto, a partir desse estudo consideram-se estabelecidas a equivalência conceitual, de itens e operacional, admitindo que os hábitos alimentares na população brasileira de regiões industrializadas, em muito se assemelham aos de países desenvolvidos de cultura ocidental. Além disso, foram respeitados o formato do instrumento original e o modo de aplicação. Quanto à equivalência semântica, as maiores dificuldades na tradução se relacionaram à forma coloquial e às expressões idiomáticas empregadas no instrumento original. Esses problemas foram resolvidos por consenso no processo de tradução e adaptação, muitas vezes, priorizando-se a adaptação à cultura brasileira, em detrimento da equivalência semântica.

Além do processo de tradução e retradução do instrumento original, foi realizada uma avaliação semântica em associação com uma interlocução com profissionais da área estudada e da população-alvo, sem isto a adaptação do instrumento perde em termos de significado geral,

Coeficiente alfa de Cronbach dos 14 itens da escala e na reanálise dos itens retirando a questão número 7 ( $n=100)$.

\section{Questões}

Valor de alfa

14 questões 13 questões *
1. Normalmente, como é o seu apetite de manhã?

2. Normalmente, quando você come pela primeira vez ao dia?

3. Depois do jantar, até a hora de dormir, você tem compulsões ou desejos de fazer lanches?

4. O quanto você consegue controlar a sua alimentação entre a janta e a hora de ir deitar?

5. Em relação à sua ingestão diária de alimentos, qual a quantidade que você costuma consumir depois de jantar?

6. Você costumeiramente se sente triste, deprimido ou um lixo?

7. Quando você se sente triste, em qual parte do dia seu humor é pior:

8. Com que freqüência você tem dificuldades para pegar no sono?

9. Quantas vezes você acorda pelo menos uma vez no meio da noite (por outra razão que não seja usar o banheiro)?

10. Você tem compulsões ou desejos de fazer lanches quando acorda à noite?

11. Quando você acorda à noite, você precisa comer para voltar a dormir?

12. Quando você acorda no meio da noite, quantas vezes você lancha?

13. Qual o seu nível de consciência quando você lancha no meio da noite?

14. Qual o nível de controle sobre sua alimentação enquanto você está acordado à noite?

Coeficiente alfa geral da escala

\section{0,84}

0,82

0,79

0,76

0,76

0,76

0,80

0,81

0,80

0,77

0,82

0,82

0,76

0,78

0,74

0,74

0,74

0,74

0,73

0,78
$-$

0,81

0,82

0,78

0,78

0,78

0,78

0,78

0,82

* A questão removida foi a no. 7 . 
limitando o instrumento ao significado referencial 23 . Todavia, outras etapas, que possam assegurar outras propriedades psicométricas e valores diagnósticos (incluindo sujeitos com diferentes níveis de escolaridade e distintos estratos sociais), em diferentes populações, ainda precisam ser desenvolvidas em estudos vindouros. Os itens 9, 12 e 13 obtiveram a menor média de clareza na escala analógico-visual, provavelmente, por avaliarem comportamentos que ocorrem à noite, período em que o nível de consciência pode estar diminuído. Além disso, a questão 13 aborda a consciência de forma dúbia, por não deixar claro se está relacionada ao estado mental ou ao nível de crítica do comportamento.

A escala mostrou adequada consistência interna (alfa de Cronbach $=0,78$ ) 30 e evidenciou melhor adequação psicométrica ao ser retirada a questão que avalia alteração do humor durante o dia (alfa de Cronbach $=0,82$ ). O incremento no coeficiente alfa de Cronbach sugere que na versão brasileira esse item deva ser examinado com maior cuidado, necessitando de novos estudos para considerar sua modificação, substituição ou exclusão. Ao serem comparados os resultados obtidos pelo coeficiente de fidedignidade de Cronbach, evidenciam que a escala total da adaptação brasileira apresentou consistência interna satisfatória, pois quando o coeficiente for acima de 0,9 pode sugerir a presença de itens redundantes, e abaixo de 0,70 pode refletir baixa consistência interna 30,31,32.

Outro aspecto a ser salientado é o alto nível de escolaridade da nossa amostra, pois estudos prévios têm demonstrado que o nível educacional influencia os desfechos em saúde e hábitos de vida aferidos por medidas de auto-relato $23,24,25,33,34$. Isso pode ter afetado a compreensão das perguntas e, conseqüentemente, as respostas. Como a escolaridade reflete a situação sócio-econômica de maneira muito próxima, o alto nível de escolaridade desses sujeitos sugere que pertençam a uma classe sócio-economicamente mais favorecida do que a população brasileira em geral, com maior habilidade cognitiva, maior acesso à informação e aos serviços de saúde 35 . Por conseguinte, é altamente desejável que esse instrumento seja aplicado em amostras com diferentes condições sociais, particularmente porque características dessa amostra como a homogeneidade e o alto nível educacional, que são desejáveis para aumentar a validade interna num processo inicial de validação de um instrumento de auto-relato, podem também ter influenciado a validação semântica e a confiabilidade, que serão importantes para a validade externa. Adicionam-se a esses aspectos particularidades sócio-demográficas levantadas em estudos reali- zados no Brasil, como uma relação inversa entre a prevalência de pré-obesidade e obesidade com o nível de escolaridade em pacientes do sexo feminino 36,37 e naquelas gestantes com ganho de peso excessivo ${ }^{35}$. Além disso, poder-se-ia cogitar que entram nesse escopo fatores que podem ser determinados pelo poder aquisitivo, como o acesso aos alimentos em termos quantitativos e qualitativos, fato que poderia induzir o consumo de alimentos mais calóricos em indivíduos com menor poder aquisitivo, por serem mais baratos. Esses aspectos corroboram a idéia de se realizar novos estudos em populações com diferentes níveis de escolaridade, para apreciar de modo mais detalhado a adequação deste instrumento, que poderá viabilizar estudos que explorem com maior profundidade dados de evidências recentes, que sugerem forte associação entre a síndrome do comer noturno, obesidade, diabetes do tipo II e pobre qualidade de vida $38,39,40$. Apesar disso, os resultados deste estudo de adaptação lingüística do Questionário sobre Hábitos do Comer Noturno (Tabela 2), obtido por meio de um cuidadoso processo de tradução e adaptação compatível com as recomendações atuais para este processo $21,22,23,24,41,42,43,44$, sugerem que se trata de um instrumento de fácil compreensão e uso, o que estimula o estudo da aplicação desta versão traduzida e adaptada em populações com níveis de escolaridade diversos.

\section{Conclusão}

A versão final do Questionário sobre Hábitos do Comer Noturno foi aplicada em pacientes de clínica nutricional, que mostraram bom nível de compreensão, sugerindo bom potencial para uso em estudos vindouros, pois os dados obtidos no processo de tradução e adaptação, bem como a confiabilidade do instrumento, sugerem que este questionário pode ser um instrumento de screening sobre hábitos alimentares noturnos por ser de auto-relato, de fácil compreensão e aplicação, e, que demanda curto espaço de tempo para ser respondido. No entanto, este é um estudo que inicia o processo de validação, sendo necessário aplicá-lo em outras populações. Além disso, é instrumento de baixo custo que avalia hábitos alimentares, tema fortemente relacionado ao processo saúde-doença, o que poderá permitir a avaliação de hábitos alimentares noturnos como fator associado à obesidade e diabetes em estudos populacionais. 


\section{Resumo}

O objetivo deste estudo foi traduzir e adaptar o conteúdo do Night Eating Questionnaire e avaliar a confiabilidade da versão em português. O estudo envolveu duas fases. A primeira constituiu-se da: (1) tradução; (2) retradução para o Inglês; (3) correção e adaptação da semântica; (4) validação do conteúdo; e (5) avaliação da clareza do questionário por meio de escalas analógico-visuais de $10 \mathrm{~cm}$ com trinta adultos de uma clínica de suporte nutricional. Na segunda, avaliou-se a confiabilidade com 100 sujeitos, cujo perfil foi similar ao da primeira etapa. Na quinta etapa, a compreensão do instrumento aferida pela escalas analógicovisuais teve média de $8,20 \pm 1,55 \mathrm{~cm}$. Este instrumento demonstrou consistência interna satisfatória, com um coeficiente geral alfa de Cronbach $=0,78$. A versão mostrou ser de fácil compreensão, obtendo-se adequada validação semântica e de consistência. Isso sugere que o instrumento pode ser adequado para screening da síndrome do comer noturno. No entanto, é necessário avaliar as características psicométricas deste instrumento em amostra com diferentes níveis sociais $e$ educacionais.

Ritmo Circadiano; Cronobiologia; Hábitos Alimentares

\section{Referências}

1. Turner RW. Coronary heart disease. The size and nature of the problem. Postgrad Med J 1980; 56:538-47.

2. Zimmet PZ, King HO, Björntorp SP. Obesity, hypertension, carbohydrate disorders and the risk of chronic diseases. Is there any epidemiological evidence for integrated prevention programmers? Med J Aust 1986; 145:256-9, 262.

3. Martins IS, Coelho LT, Mazzilli RN, Singer JM, Souza CU, Antonieto Júnior AE, et al. Atherosclerotic cardiovascular diseases, dyslipidemia, hypertension, obesity, and diabetes mellitus in a population of the metropolitan area of southeastern Brazil. IResearch methodology. Rev Saúde Pública 1993; 27:250-61.

4. Gooley JJ, Schomer A, Saper CB. The dorsomedial hypothalamic nucleus is critical for the expression of food-entrainable circadian rhythms. Nat Neurosci 2006; 9:398-407.

5. Ko GT, Chan JC, Chan AW, Wong PT, Hui SS, Tong $\mathrm{SD}$, et al. Association between sleeping hours, working hours and obesity in Hong Kong Chinese: the 'better health for better Hong Kong' health promotion campaign. Int J Obes 2007; 31:254-60.

6. Qin LQ, Li J, Wang Y, Wang J, Xu JY, Kaneko T. The effects of nocturnal life on endocrine circadian patterns in healthy adults. Life Science 2003; 73:2467-75.

7. Morgan L, Arendt J, Owens D, Folkard S, Hampton S, Deacon S, et al. Effects of the endogenous clock and sleep time on melatonin, insulin, glucose and lipid metabolism. J Endocrinol 1998; 157:443-51.

\section{Colaboradores}

Todos os autores participaram da elaboração do projeto, execução do estudo, análise dos dados e elaboração do artigo.

\section{Agradecimentos}

Kelly Allison, autora do Night Eating Questionnaire, pelas valiosas contribuições no processo de tradução e adaptação para a Língua Portuguesa.
8. Spiegel K, Leproult R, Van Cauter E. Impact of sleep debt on metabolic and endocrine function. Lancet 1999; 354:1435-9.

9. Allison KC, Stunkard AJ, Thier SL. Overcoming night eating syndrome: a step-by-step guide to breaking the cycle. Oakland: New Harbinger; 2004.

10. Hidalgo M, Souza C, Zanette C, Nunes P, Voltolini S, Pedrotti M, et al. Revisão sobre cronobiologia e aspectos cognitivo-comportamentais relacionados a cronotipos. Rev Psiquiatr Rio Gd Sul 2002; 25:174-81.

11. Waterhouse J, Buckley P, Edwards B, Reilly T. Measurement of, and some reasons for, differences in eating habits between night and day workers. Chronobiol Int 2003; 20:1075-92.

12. Harthoorn LF, Sane A, Nethe M, Van Heerikhuize JJ. Multi-transcriptional profiling of melanin-concentrating hormone and orexin-containing neurons. Cell Mol Neurobiol 2005; 25:1209-23.

13. Shea SA, Hilton MF, Orlova C, Ayers RT, Mantzoros CS. Independent circadian and sleep/wake regulation of adipokines and glucose in humans. J Clin Endocrinol Metab 2005; 90:2537-44.

14. Birketvedt GS, Florholmen J, Sundsfjord J, Osterud B, Dinges D, Bilker W, et al. Behavioral and neuroendocrine characteristics of the night-eating syndrome. JAMA 1999; 282:657-63.

15. Birketvedt GS, Sundsfjord J, Florholmen JR. Hypothalamic-pituitary-adrenal axis in the night eating syndrome. Am J Physiol Endocrinol Metab 2002; 282:E366-9. 
16. Allison KC, Wadden TA, Sarwer DB, Fabricatore AN, Crerand CE, Gibbons LM, et al. Night eating syndrome and binge eating disorder among persons seeking bariatric surgery: prevalence and related features. Surg Obes Relat Dis 2006; 2:153-8.

17. Stunkard A. Two eating disorders: binge eating disorder and the night eating syndrome. Appetite 2000; 34:333-4

18. Stunkard AJ, Allison KC. Binge eating disorder: disorder or marker? Int J Eat Disord 2003; 34 Suppl: S107-16.

19. Allison KC, Grilo CM, Masheb RM, Stunkard AJ. Binge eating disorder and night eating syndrome: a comparative study of disordered eating. J Consult Clin Psychol 2005; 73:1107-15.

20. O’Reardon JP, Stunkard AJ, Allison KC. Clinical trial of sertraline in the treatment of night eating syndrome. Int J Eat Disord 2004; 35:16-26.

21. Beaton DE, Bombardier C, Guillemin F, Ferraz MB. Guidelines for the process of cross-cultural adaptation of self-report measures. Spine 2000; 25:3186-91.

22. Freitas S, Lopes CS, Coutinho W, Appolinario JC. Tradução e adaptação para o português da Escala de Compulsão Alimentar Periódica. Rev Bras Psiquiatr 2001; 23:215-20.

23. Grassi-Oliveira R, Stein LM, Pezzi JC. Translation and content validation of the Childhood Trauma Questionnaire into Portuguese language. Rev Saúde Pública 2006; 40:249-55.

24. Reichenheim ME, Moraes CL, Hasselmann MH. Semantic equivalence of the Portuguese version of the Abuse Assessment Screen tool used for the screening of violence against pregnant women. Rev Saúde Pública 2000; 34:610-6.

25. Pasquali L. Princípios de elaboração de escalas psicológicas. In: Gorestein C, Andrade LHSG, Zuardi AW, organizadores. Escalas de avaliação clínica em psiquiatria e psicofarmacologia. São Paulo: Lemos Editorial; 2000. p. 15-21.

26. Jorge MR. Adaptação transcultural de instrumentos de pesquisa em saúde mental. In: Gorestein C, Andrade LHSG, Zuardi AW, organizadores. Escalas de avaliação clínica em psiquiatria e psicofarmacologia. São Paulo: Lemos Editorial; 2000. p. 53-9.

27. Clark P, Levielle P, Martinez H. Learning from pain scales: patient perspective. J Rheumatol 2003; 30:1584-8.

28. Creamer P, Lethbridge-Cejku M, Hochberg MC. Determinants of pain severity in knee osteoarthritis: effect of demographic and psychosocial variables using 3 pain measures. J Rheumatol 1999; 26:1785-92.

29. Rowland D, Arkkelin D, Crisler L. Computer-based data analysis: using SPSS in the Social and Sciences. Chicago: Wardsworth Publishing Co.; 1991.

30. Cortina JM. What is coefficient alpha? An examination of theory and applications. J Appl Psychol 1993; 78:98-104.

31. Pedroso RS, Oliveira MS, Moraes JFD. Tradução, adaptação e validação da versão brasileira da escala Marijuana Expectancy Questionnaire. Cad Saúde Pública 2007; 23:63-73.
32. Silva KS, Coutinho ESF. Escala de apoio social aplicada a uma população de gestantes: confiabilidade teste-reteste e estrutura de concordância dos itens. Cad Saúde Pública 2005; 21:979-83.

33. Byrne B. The general/academic self-concept nomological network: a review of construct validation research. Rev Educ Res 1984; 54:427-56.

34. Chor D, Faerstein E, Alves MG, Lopes CS. How reproducible is self-reported information on exposure to smoking, drinking, and dietary patterns? Evidence among Brazilian adults in the Pro-Saude Study. São Paulo Med J 2003; 121:63-6.

35. Monteiro CA, Conde WL, Castro IRR. A tendência cambiante da relação entre escolaridade e risco de obesidade no Brasil (1975-1997). Cad Saúde Pública 2003; 19 Suppl 1:S67-75.

36. Bossan FM, Anjos LA, Vasconcellos MTL, Wahrlich V. Nutritional status of the adult population in $\mathrm{Ni}$ terói, Rio de Janeiro, Brazil: the Nutrition, Physical Activity, and Health Survey. Cad Saúde Pública 2007; 23:1867-76.

37. Andreto LM, Souza AI, Figueroa JN, Cabral-Filho JE. Fatores associados ao ganho ponderal excessivo em gestantes atendidas em um serviço público de pré-natal na Cidade de Recife, Pernambuco, Brasil. Cad Saúde Pública 2006; 22:2401-9.

38. Yun S, Zhu BP, BlackW, Brownson RC. Comparison of national estimates of obesity prevalence from the behavioral risk factor surveillance system and the National Health and Nutrition Examination Survey. Int J Obes 2006; 30:164-70.

39. Colles SL, Dixon JB, O’Brien PE. Night eating syndrome and nocturnal snacking: association with obesity, binge eating and psychological distress. Int J Obes (Lond) 2007; 31:1722-30.

40. Allison KC, Crow SJ, Reeves RR, West DS, Foreyt JP, Dilillo VG, et al. Binge eating disorder and night eating syndrome in adults with type 2 diabetes. Obesity 2007; 15:1287-93.

41. Subramanian SV, Kim D, Kawachi I. Covariation in the socioeconomic determinants of self rated health and happiness: a multivariate multilevel analysis of individuals and communities in the USA. J Epidemiol Community Health 2005; 59: 664-9.

42. Gandek B, Ware Jr. JE. Methods for validating and norming translations of health status questionnaires: the IQOLA Project approach. International Quality of Life Assessment. J Clin Epidemiol 1998; 51:953-9.

43. Bullinger M, Alonso J, Apolone G, Leplege A, Sullivan M, Wood-Dauphinee S, et al. Translating health status questionnaires and evaluating their quality: the IQOLA Project approach. International Quality of Life Assessment. J Clin Epidemiol 1998; 51:913-23.

44. Herdman M, Fox-Rushby J, Badia X. 'Equivalence' and the translation and adaptation of health-related quality of life questionnaires. Qual Life Res 1997; 6:237-47.

Recebido em 14/Mai/2007

Versão final reapresentada em 05/Nov/2007 Aprovado em 13/Nov/2007 